\title{
DE HISPANIA A AL-ANDALUS. DINÁMICA DE CAMBIO CULTURAL A LA LUZ DE LA ARQUEOLOGÍA
}

\author{
FROM HISPANIA TO AL-ANDALUS. CULTURAL CHANGES ACCORDING TO ARCHAEOLOGY
}

\author{
SONIA GUTIÉRREZ LLORET \\ Universidad de Alicante
}

El número 27 de la revista Lucentum incluye una serie de artículos sobre la lectura arqueológica del cambio cultural en la formación de al-Andalus. Son los resultados de un seminario celebrado en la Universidad de Alicante, cuya edición y publicación se debe al encuentro de varias voluntades personales y colectivas de las que creo obligado dar cuenta en estas líneas.

En primer lugar está la voluntad de la propia revista, Lucentum, creada al tiempo que la misma Universidad de Alicante, como medio de expresión de las Áreas de Prehistoria, Arqueología e Historia Antigua, y que en estos 26 años de vida ha sido el principal vehículo de transferencia del conocimiento científico generado por la investigación histórica y arqueológica de nuestro ámbito. La revista a lo largo de estos años y como reflejo de la evolución conceptual de las disciplinas que se difunden a través de ella, se ha abierto a las innovaciones, perspectivas y planteamientos que han ido modelando unos contenidos académicos inicialmente centrados en la antigüedad, en beneficio de una visión diacrónica más amplia que acoge también el aporte de la arqueología a la reflexión histórica de las sociedades postclásicas.

En segundo lugar está la voluntad de la Universidad de Alicante, dispuesta a apoyar la transmisión del conocimiento científico a través de las Ayudas de Extensión Universitaria y Cultura, que permitió organizar el Seminario de arqueología «De Hispania a al-Andalus. Dinámica de cambio cultural a la luz de la Arqueología», celebrado en la Universidad de Alicante los días 9 y 10 de abril de 2008. En el desarrollo de este proyecto quiero destacar la colaboración de Gabriel Lara Vives, Julia Sarabia Bautista y Victor Cañavate Castejón, becarios y miembros del equipo científico del Tolmo de Minateda, que se ocuparon conmigo del diseño del seminario y de la recopilación de los trabajos.

El seminario pretendía favorecer el intercambio de experiencias entre diferentes equipos de investigación centrados en el reconocimiento material de la islamización temprana y establecer un foro de discusión científica abierto a los miembros de la comunidad universitaria. Para ello se propusieron una decena de intervenciones que repasaron las más innovadoras y recientes aportaciones en el campo de la Alta Edad Media en el sudeste de al-Andalus, en confrontación con los resultados de los proyectos de investigación que realiza la Universidad de Alicante sobre dicha temática. De esta forma se daba cabida tanto a trabajos que realizaban miembros de la comunidad universitaria alicantina en las Comunidades Castellano-Manchega (Parques Arqueológicos del Tolmo de Minateda en Albacete o Segobriga en Cuenca) y Valenciana (el conjunto del Molón de Camporrobles en Valencia o el Castellar de Morera en Elche, en este caso en colaboración con el MARQ), como a investigaciones novedosas desarrolladas desde otros marcos institucionales que aportaban indicios fundamentales para comprender el proceso histórico de la islamización (caso de las maqbaras tempranas de Pamplona o del Tossal de Manises o de yacimientos como el Cabezo Pardo o el Castellar de Alcoi). El coloquio contó además con tres intervenciones marco destinadas a centrar la problemática del poblamiento («El inicio de la torre residencial en al-Andalus», a cargo de Manuel Acién de la Universidad de Málaga) y a confrontarla con dos ejemplos urbanos próximos y bien documentados «Cartagena en los primeros siglos de presencia islámica», a cargo de Martín Guillermo del Museo del Teatro Romano de Cartagena, y «Madina Balansiya entre los siglos VIII-X», a cargo de Javier Martí y Pepa Pascual del SIAM de Valencia.

El seminario puso en evidencia el potencial informativo que la arqueología aportaba en la comprensión del proceso de islamización, plasmando por otro lado la vitalidad de la disciplina en un momento en que la arqueología islámica, verdadero motor de innovación de la arqueología medieval española en las últimas décadas, parece sufrir un cierto estancamiento frente al dinamismo de la investigación en la Alta Edad Media protofeudal y «europea». El revelador volumen de trabajos en curso que afloraron a raíz de la reunión y que 
no habían tenido cabida en la misma, nos llevaron al convencimiento de que era necesario divulgar dichos progresos y presentarlos a la comunidad científica, acelerando un proceso de transmisión del conocimiento que en ocasiones no encuentra un medio de difusión riguroso e inmediato. Quiero agradecer desde aquí la disponibilidad de la revista Lucentum a la hora de asumir este papel y su comprensión ante un volumen final de trabajos que desbordó las previsiones iniciales más optimistas.

El conjunto de trabajos que viene a continuación ha quedado configurado por once artículos que analizan diversas problemáticas a partir de asentamientos, espacios funerarios y elementos de cultura material, centrados preferentemente en la problemática del Emirato y el Califato. Lo inicia El Tolmo de Minateda, uno de los proyectos emblemáticos de la Universidad de Alicante sobre la transición del mundo visigodo al islámico, con dos trabajos específicos firmados por Víctor Cañavate y Julia Sarabia, dedicados respectivamente a la aplicación innovadora del análisis arqueotectónico a la arquitectura doméstica emiral y al reempleo en la construcción de dichos ambientes domésticos. Debo señalar que la omisión de un trabajo de reflexión general sobre la problemática de la ciudad emiral del Tolmo ha sido consciente y responde al convencimiento de que un equipo de investigación como el nuestro debe amparar también la investigación tutelada pero personal, de los miembros más jóvenes del equipo. Los estudios del Molón de Camporrobles (a cargo de Alberto Lorrio y M $\mathrm{M}^{\mathrm{a}}$ Dolores Sánchez de Prado) y del Cabezo Pardo (de Juan A. López Padilla y Teresa Ximénez de Embún) ponen en evidencia cómo proyectos rigurosos, concebidos para estudiar aspectos de la prehistoria y protohistoria, pueden y deben producir reflexiones históricas de cualidad sobre un problema histórico distinto del inicialmente planteado, aportando en ambos casos informaciones valiosísimas sobre la islamización. El Castellar de Morera, estudiado por José L. Menéndez, Pierre Guichard y yo misma, refleja los primeros resultados de la investigación sobre un yacimiento invisible, que se materializa ahora como un importante asentamiento fortificado que debe ser comprendido a la luz de las convulsiones de la primera fitna y de la consolidación del Califato.

Un contenido marcadamente cerámológico ofrecen los trabajos sobre el Castellar de Alcoi de Germán Pérez Botí, y sobre la ocupación emiral de Segobriga a cargo de Daniel Sanfeliu y Rosario Cebrián. El primero aborda el estudio en profundidad de los materiales cerámicos de un yacimiento fechado generalmente entre los siglos X y XI y fundamental en la historiografía alicantina, el Castellar de Alcoi, excavado a finales del los años setenta con los criterios de artificialidad estratigráfica propios de la época, proponiendo para el mismo un eventual origen emiral que habrá de ser contrastado en futuras excavaciones estratigráficas. El segundo presenta por vez primera las evidencias arqueológicas de una fase de ocupación emiral constatada en diversos lugares de la ciudad romana de Segobriga, sobre los niveles visigodos, reforzando la imagen de una larga secuencia de ocupación urbana a menudo científicamente difuminada por la esplendorosa materialidad de la fase romana.

El ámbito funerario viene ejemplificado en el estudio de la maqbara del Tossal de Manises, realizado por Manuel Olcina, Eva Tendero y Antonio Guilabert, que representa la última de las ocupaciones constatadas en el solar de la ciudad ibero-romana de Lucentum y cuya cronología temprana permite plantear el problema del poblamiento en la zona en relación al origen de la madina de Laqant (Alicante). El estudio de los restos óseos hallados en las necrópolis ha abierto interesantes perspectivas de análisis paleodemográficos, paleonutricionales y paleopatológicos, plasmados en el estudio del propio Tossal realizado por Consuelo Roca de Togores, y en el interesante análisis de cinco gestantes procedentes de contextos altomedievales navarros realizado por $\mathrm{M}^{\mathrm{a}}$ Paz de Miguel, quien estudia además el importante hallazgo reciente de la maqbara de Pamplona. Por fin, Carolina Doménech replantea el problema de los alifatos árabes inscritos sobre hueso, de finalidad en apariencia docente, a partir del hallazgo contextualizado de una de estas piezas en el casco antiguo de Alicante, así como sus supuestas cronologías tempranas que a la luz de las contextualizaciones estratigráficas otorgan amplios márgenes entre los siglos IX y XII.

Creo que la publicación conjunta de estos trabajos constituye un buen observatorio del dinamismo y la potencia explicativa de la arqueología en la discusión histórica sobre la formación de la sociedad islámica en al-Andalus y abre interesantes expectativas de las que la revista Lucentum quiere dar cuenta.

\author{
Profa. Dra. Sonia Gutiérrez Lloret \\ Área de Arqueología \\ Dpto. de Prehistoria, Arqueología, $\mathrm{H}^{\mathrm{a}}$ Antigua, \\ Filología Griega y Filología Latina \\ Facultad de Filosofía y Letras \\ Universidad de Alicante \\ 03080 Alicante \\ sonia.gutierrez@ua.es
}

